\title{
A randomized, double-blind, placebo-controlled phase 2 pilot trial evaluating a novel, vaginal softgel capsule containing solubilized estradiol
}

\author{
James H. Pickar, MD, ${ }^{1}$ Julia M. Amadio, ${ }^{2}$ John M. Hill, MD, ${ }^{3}$ Brian A. Bernick, MD, ${ }^{2}$ \\ and Sebastian Mirkin, $M D^{2}$
}

\begin{abstract}
Objective: The aim of the study was to evaluate the safety and efficacy of vaginal estradiol $\left(\mathrm{E}_{2}\right)$ softgel capsules for moderate-to-severe symptoms of vulvar and vaginal atrophy (VVA). Previous phase 1 studies showed lower systemic estrogen concentrations with this softgel capsule compared with an approved low-dose vaginal $\mathrm{E}_{2}$ tablet.

Methods: In this randomized, double-blind, placebo-controlled phase 2 study, 50 postmenopausal women (aged 40-75 y) with at least1 moderate-to-severe VVA symptom received $10 \mu \mathrm{g}$ vaginal $\mathrm{E}_{2}$ softgel capsules or placebo daily for 14 days. Changes from baseline in vaginal maturation index, investigator's assessment of vaginal mucosa (secretions, epithelial integrity, epithelial surface thickness, color), vaginal $\mathrm{pH}$, and most bothersome symptom were assessed. Adverse events were evaluated.

Results: Compared with placebo, the percentage of superficial (35.2 percentage points [pp] vs $8.75 \mathrm{pp}$; $P=0.0002)$ and intermediate $(18.7 \mathrm{pp}$ vs $-3.54 \mathrm{pp} ; P=0.0017)$ cells increased from baseline significantly more with vaginal $\mathrm{E}_{2}$ capsules, and parabasal cells decreased significantly more $(-54.4 \mathrm{pp}$ vs $-4.80 \mathrm{pp} ; P<0.0001)$. Vaginal $\mathrm{pH}$ decreased significantly more with vaginal $\mathrm{E}_{2}$ capsules $(-0.974$ vs $-0.339 ; P=0.0002)$. Decreases in severity of atrophic effects on vaginal epithelial integrity $(-0.342$ vs $0.176 ; P=0.0001)$ and secretions $(-0.643$ vs $-0.274 ; P=0.0401)$ were significantly greater with vaginal $\mathrm{E}_{2}$ capsules vs placebo. There was no statistical difference in most bothersome symptom severity change from baseline. No serious adverse events were reported.

Conclusions: Vaginal $\mathrm{E}_{2}$ softgel capsules are a safe, effective, local treatment option for postmenopausal women with moderate-to-severe VVA, with lower systemic estrogen absorption than currently available intravaginal treatments.
\end{abstract}

Key Words: Estradiol - Estrogen therapy - Menopause - Vaginal atrophy.

$\mathrm{M}$ enopause is marked by a decline in endogenous estrogen, which causes atrophic changes to occur in the genital tract. Over time, the tissue lining the

Received June 18, 2015; revised and accepted November 4, 2015.

From the ${ }^{1}$ Columbia University Medical Center, New York, NY; ${ }^{2}$ TherapeuticsMD, Boca Raton, FL; and ${ }^{3}$ Avail Clinical Research LLC, DeLand, FL.

Funding/support: TherapeuticsMD sponsored the study and funded the medical writing support provided by Chastity Bradley, $\mathrm{PhD}$, and Jolene Mason, $\mathrm{PhD}$, of Precise Publications, LLC.

Financial disclosure/conflicts of interest: Dr Pickar was formerly an employee of Wyeth Research; has received consultant fees from Wyeth/ Pfizer, Besins Healthcare, Shionogi Inc, Metagenics, and TherapeuticsMD; and has stock options with TherapeuticsMD (but was not paid for his role as an author on this paper). Dr Hill declares no conflicts of interest. Dr Bernick, Dr Mirkin, and Ms Amadio are employees of TherapeuticsMD.

Address correspondence to: James H. Pickar, MD, Columbia University Medical Center, PO Box 415, Bulger, PA 15019.

E-mail: jhpickar@me.com

This is an open-access article distributed under the terms of the Creative Commons Attribution-Non Commercial-No Derivatives License 4.0 (CCBY-NC-ND), where it is permissible to download and share the work provided it is properly cited. The work cannot be changed in any way or used commercially. vagina becomes thinner, drier, and less elastic, resulting in vulvar and vaginal atrophy (VVA; also known as genitourinary syndrome of menopause). ${ }^{1}$ VVA is generally accompanied by symptoms such as vaginal dryness, irritation, itching, dysuria, and vaginal pain or bleeding associated with sexual activity ${ }^{2,3}$; however, early, sustained treatment can preclude irreversible declines in vaginal health. ${ }^{4}$ Up to 32 million women in the United States currently are suffering from symptoms of VVA, ${ }^{5,6}$ and more than $85 \%$ of this population currently receives no prescription therapy for the condition. ${ }^{7}$ Effective treatments designed to help relieve the pain and discomfort associated with VVA symptoms will improve the quality of life for many women.

The North American Menopause Society and the International Menopause Society recommend local vaginal estrogens when menopausal symptoms are limited to VVA., ${ }^{4,8}$ Despite the relative safety and efficacy of local vaginal treatments, consumers remain dissatisfied with the currently available local treatment options. ${ }^{7}$ Vaginal creams approved in the 1980s are antiquated. They require an applicator, can be unsanitary and inconvenient for women to apply, and dosing can be complicated and confusing. ${ }^{3}$ The creams can be messy 
and cause excessive discharge, leading some women to alternatively choose vaginal tablets or rings. ${ }^{3,10}$ Vaginal tablets currently on the market, however, can be difficult to use, may not fully dissolve for days, and may be inconvenient, in addition to concerns of overall treatment efficacy. Likewise, the vaginal ring may be difficult to insert and remove, ${ }^{3}$ may change position or dislodge, ${ }^{9}$ and has been shown to release an initial burst of systemic estradiol $\left(\mathrm{E}_{2}\right) .{ }^{11}$

A new softgel vaginal capsule (VagiCap) that contains solubilized $17 \beta-\mathrm{E}_{2}$, known as TX-004HR (TherapeuticsMD, Inc, Boca Raton, FL), was designed to treat moderate-tosevere symptoms of VVA in postmenopausal women. Pharmacokinetic studies have demonstrated lower systemic estrogen exposure with TX-004HR as compared with equivalent doses of the vaginal $E_{2}$ tablet (Vagifem, Novo Nordisk, Plainsboro, NJ). ${ }^{12}$ This lower systemic exposure of $\mathrm{E}_{2}$ supports the call of the Working Group on Women's Health and Well-Being in Menopause to modify the boxed warning on labels and package inserts for low-dose vaginal estrogen. ${ }^{13}$ The warning included in the black box on low-dose vaginal estrogens is based on extrapolations from trials of systemic estrogen or combination estrogen-progestin hormone preparations. ${ }^{13}$ The Working Group proposes to distinguish that the relevance of findings of risks associated with systemic estrogen administration is not applicable to low-dose vaginal estrogen preparations. ${ }^{13}$ This clarity is likely to increase use and compliance, resulting in substantial vaginal health benefits to a broader population.

If approved, TX-004HR, as a vaginal $\mathrm{E}_{2}$ softgel capsule, would fill an unmet need for a more user-friendly, modern product, considering that most of the available products were approved more than 20 years ago. TX-004HR is expected to provide improved ease of vaginal administration without the need of an applicator and minimize vaginal discharge after administration. TX-004HR may also provide a more effective dosage form with improved efficacy, early onset of action, and patient compliance, as well as lower systemic estrogen levels than currently available products. The phase 3 REJOICE trial evaluated the safety and efficacy of TX$004 \mathrm{HR}$ vaginal $\mathrm{E}_{2}$ softgel capsules at 4,10 , and $25 \mu \mathrm{g}$ doses in the United States and Canada.

The overall objective of this phase 2 pilot study was to evaluate the safety and efficacy of $10-\mu \mathrm{g}$ TX-004HR vaginal $\mathrm{E}_{2}$ softgel capsules, when compared with placebo, as a treatment for postmenopausal women suffering from moderate-tosevere symptoms of VVA.

\section{METHODS}

\section{Study design and drug administration}

In this single-center, double-blind, placebo-controlled phase 2 pilot trial, eligible women were randomized 1:1 to either $10 \mu \mathrm{g}$ TX-004HR vaginal $\mathrm{E}_{2}$ softgel capsules or matching placebo vaginal softgel capsules (TherapeuticsMD) administered intravaginally once-daily for 14 days. A reproducible, computer-generated block randomization schedule (produced in SAS v. 9.1.3, PLAN procedure) was used to randomize the women to the study groups. Biostudy Solutions, LLC generated the assignment codes, which were locked in a room with restricted access. The packaging, labeling, appearance, and route of administration of TX004HR and placebo were identical, and all persons participating in the study were blinded to the treatment each woman received. The blind was maintained until study completion.

On day 1, participants completed a questionnaire that assessed VVA symptoms and specified their most bothersome symptom (MBS), and the investigator assessed the appearance of the vaginal mucosa (secretions, color, epithelial integrity, and epithelial surface thickness; see Table 1). Women self-administered their first dose of study medication at the clinical site under supervision, and capsule disintegration was assessed after 6 hours. During screening, vaginal smears were collected to evaluate vaginal $\mathrm{pH}$ and cytology

TABLE 1. Participant demographics and baseline characteristics

\begin{tabular}{|c|c|c|}
\hline Parameter & Vaginal $\mathrm{E}_{2}$ softgel $(\mathrm{N}=24)$ & Placebo $(\mathrm{N}=24)$ \\
\hline Age, $y$ & $62.4 \pm 5.7$ & $62.6 \pm 7.3$ \\
\hline BMI, $\mathrm{kg} / \mathrm{m}^{2}$ & $26.4 \pm 3.9$ & $27.4 \pm 3.4$ \\
\hline Estradiol, $\mathrm{pg} / \mathrm{mL}^{a}$ & $8.38 \pm 9.54$ & $6.96 \pm 7.56$ \\
\hline Parabasal cells, $\%$ & $62.5 \pm 40.1$ & $64.6 \pm 38.8$ \\
\hline Superficial cells, $\%$ & $1.7 \pm 2.4$ & $0.2 \pm 1.0$ \\
\hline Intermediate cells, $\%$ & $35.8 \pm 38.3$ & $35.2 \pm 38.4$ \\
\hline $\mathrm{pH}$ & 6.00 & 6.13 \\
\hline MBS (mean severity score ${ }^{b}$ ) & 2.52 & 2.58 \\
\hline \multicolumn{3}{|l|}{ VVA symptoms (mean severity score ${ }^{b}$ ) } \\
\hline Vaginal dryness & 2.292 & 2.375 \\
\hline Vaginal and/or vulvar irritation/itching & 0.875 & 1.333 \\
\hline Pain, burning, or stinging when urinating & 0.583 & 0.625 \\
\hline \multicolumn{3}{|l|}{ Sexually active women ${ }^{c}$} \\
\hline Vaginal pain with sexual activity (mean severity score) & 2.083 & 2.333 \\
\hline Vaginal bleeding with sexual activity, $\%$ & 25 & 33.33 \\
\hline
\end{tabular}

Data are presented as the mean $\pm \mathrm{SD}$, except where noted.

BMI, body mass index.

${ }^{a}$ Values below $10 \mathrm{pg} / \mathrm{mL}$ were set to 0.0 .

${ }^{b}$ Subjectively assessed as $0=$ none, $1=$ mild, $2=$ moderate, and $3=$ severe

${ }^{c} \mathrm{n}=12$ women for each study arm sexually active at baseline. 
(vaginal maturation index [VMI]). Participants were provided with a diary and instructed to record dosing dates and times for the 2-week study period. On day 15, VVA symptoms (participant assessment via questionnaire), vaginal mucosa, cytology, $\mathrm{pH}$, and capsule disintegration (investigator assessment) were evaluated. Diaries were collected and adverse events (AEs) were assessed on days 8 and $15 . \mathrm{E}_{2}$ levels were tested during screening at a local laboratory using a lower limit of quantification of less than $10 \mathrm{pg} / \mathrm{mL}$.

An independent institutional review board approved the protocol, and participants provided written informed consent before any study-related activities. The trial was conducted in accordance with Good Clinical Practices, US federal regulations, and the Declaration of Helsinki regarding treatment of human participants in a study.

\section{Study participants}

Postmenopausal women (40-75 y of age) were enrolled if they had a body mass index less than or equal to $34 \mathrm{~kg} / \mathrm{m}^{2}$; less than or equal to $5 \%$ superficial cells on vaginal smear cytology; a vaginal $\mathrm{pH}$ more than 5.0; an $\mathrm{E}_{2}$ level less than or equal to $50 \mathrm{pg} / \mathrm{mL}$; and at least one of the following symptoms of VVA with a moderate-to-severe severity score: vaginal dryness, vaginal pain associated with sexual activity, vaginal and/or vulvar irritation/itching, dysuria, or vaginal bleeding associated with sexual activity.

Women were excluded if they had a current or prior medical condition considered clinically significant, undiagnosed vaginal bleeding, or a current vaginal infection requiring treatment; had a contraindication to estrogen therapy (ET) or an allergy to $\mathrm{E}_{2}$; smoked at least 15 cigarettes per day; or had substance abuse in the past year. Participants could not have taken any prescription or over-the-counter drug that might interact with $E_{2}$ therapy within 28 days of screening, or used progestogen implants, vaginal or injectable ET, or vaginal or injectable estrogen-progestin therapy (EPT) within the past 3 months; transdermal ET or EPT or ET lotions or gels within the past 8 weeks; or estrogen pellets or injectable progestogen therapy within the past 6 months.

\section{Study endpoints}

Efficacy endpoints were based on the FDA's Guidance, ${ }^{14}$ and included change from baseline (screening) to day 15 in the percentage of parabasal vaginal cells, percentage of superficial vaginal cells, and percentage of intermediate vaginal cells; in vaginal $\mathrm{pH}$; and change from baseline (randomization) to day 15 in self-assessed severity of most bothersome VVA symptom (MBS). The range of MBS severity scores was 0 (none) to 3 (severe). Endpoints also included change from baseline to day 15 in investigator assessment of the vaginal mucosa (secretions, epithelial integrity, epithelial surface thickness, and color). The severity scale ranged from 0 to 3 , with 0 indicating no atrophy and 3 indicating severe atrophy.

Safety endpoints included changes in vital signs, weight, results of a physical examination (including breast and pelvic examination), and AEs.

\section{Statistical analyses}

A sample size of 50 (25 women per treatment arm) was considered adequate to estimate the magnitude and variability of efficacy measures needed to determine sample size for a future phase 3 trial. Descriptive and inferential statistical analyses were performed using SAS v.9.1.3. All statistical tests were two-sided. A $P$ value of 0.05 was considered statistically significant, with the exception that confidence intervals were based on an alpha level of 0.10 (ie, 90\% CI).

Efficacy endpoints were expressed in terms of change from baseline. For all comparisons except severity of the MBS and investigator assessments of the vaginal mucosa, the change from baseline values was evaluated using ANCOVA with baseline as the covariate, to analyze the differences between the TX-004HR vaginal $E_{2}$ softgel capsules and placebo groups.

The frequency and severity of AEs were summarized descriptively for each group. All randomized participants were included in the safety evaluation.

\section{RESULTS}

\section{Participant disposition and baseline characteristics}

Fifty postmenopausal women were enrolled in the study and randomized to either TX-004HR vaginal $\mathrm{E}_{2}$ softgel capsule $(n=24)$ or placebo $(n=26)$. Two women in the placebo group discontinued treatment; one was due to an $\mathrm{AE}$ (vulvovaginal discomfort) and the other withdrew consent.

Overall, participant demographics and baseline characteristics were not statistically different between the treatment groups (Table 1).

\section{EFFICACY}

Vaginal maturation index and $\mathbf{p H}$

The prevalence of superficial cells increased from baseline by 35.2 percentage points with TX-004HR at day 15 . This increase was significantly greater than the increase seen with placebo of 8.75 percentage points $(P=0.0002$; Fig. 1$)$. The

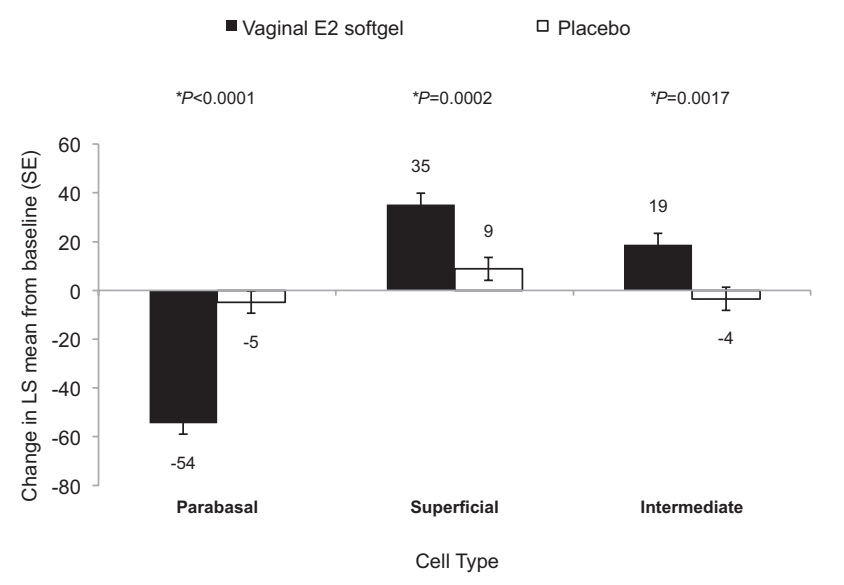

FIG. 1. Changes from baseline in the maturation index of the vaginal smear (least squares [LS] means and standard errors [SE]). ${ }^{*} P$ values represent significant differences in LS mean change from baseline to day 15 between the vaginal $E_{2}$ softgel capsule and placebo using ANCOVA with treatment as a fixed effect and baseline as a covariate. Numerical values are rounded to the nearest whole number. 
change from baseline to day 15 in the percentage of intermediate cells also increased more with TX-004HR vaginal softgel capsules (18.7 percentage points) compared with placebo (-3.54 percentage points; $P=0.0017$; Fig. 1). Accordingly, the prevalence of parabasal cells showed a significantly greater decrease from baseline to day 15 with TX-004HR than with placebo $(-54.4$ percentage points vs -4.80 percentage points; $P<0.0001$; Fig. 1 ).

Vaginal $\mathrm{pH}$ decreased significantly more from baseline to day 15 with TX-004HR $(-0.974)$ than with placebo $(-0.339$; $P=0.0002)$.

\section{VVA most bothersome symptom}

Overall, no significant difference in the decrease from baseline in severity of the MBS between treatments was found $(-1.043 \mathrm{vs}-1.042$ for TX-004HR vs placebo, respectively; $P=0.9951)$. Although no statistical difference between groups was found, the individual symptoms of dryness and dyspareunia decreased in severity by approximately 1.0 and 0.8 points versus approximately 0.7 points and 0.5 points for the $E_{2}$ softgel capsules and placebo, respectively.

\section{Investigator assessment of the vaginal mucosa}

Greater mean decreases from baseline were observed with vaginal epithelial integrity $(-0.342$ vs $0.176 ; P=0.0001)$ and vaginal secretions $(-0.643 \mathrm{vs}-0.274 ; P=0.0401)$ in the TX$004 \mathrm{HR}$ vaginal $E_{2}$ softgel capsules group compared with the placebo group (Fig. 2). No significant differences from baseline were observed for vaginal color or epithelial surface thickness between treatments.

\section{SAFETY}

Over the 14-day study period, a total of 14 (28\%) women experienced 17 AEs (13 AEs in the TX-004HR vaginal $E_{2}$ softgel group and 4 AEs in the placebo group). All AEs were

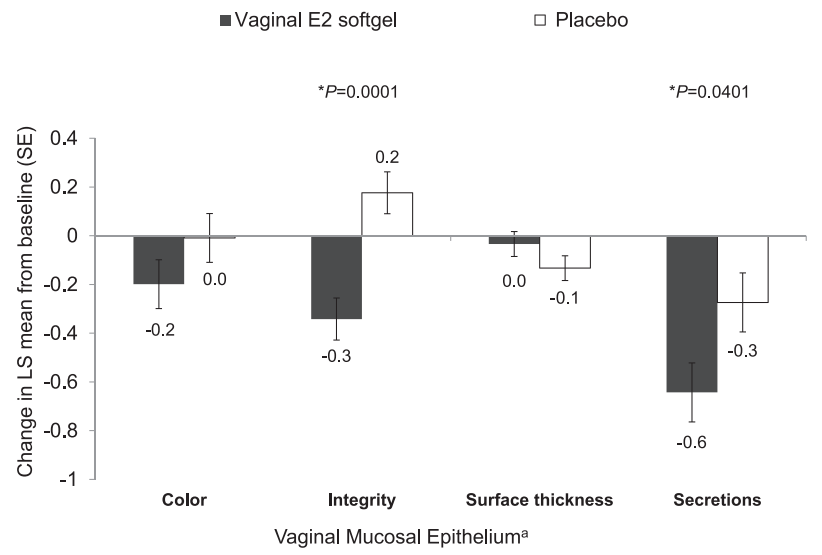

FIG. 2. Investigator's assessment of the mucosal epithelium (least squares [LS] means and standard errors [SE]). ${ }^{*} P$ values represent significant differences in LS mean change from baseline to day 15 between the vaginal $E_{2}$ softgel capsule and placebo using ANCOVA with treatment as a fixed effect and baseline as a covariate. Numerical values are rounded to the nearest tenth. ${ }^{a}$ Atrophy subjectively assessed as $0=$ none, $1=$ mild, $2=$ moderate, and $3=$ severe . mild in severity, no serious AEs were reported, and no deaths occurred during the study.

\section{DISCUSSION}

This phase 2 study showed that TX-004HR, a novel, solubilized $\mathrm{E}_{2}$ vaginal softgel capsule, was better than placebo in improving the VMI, reducing vaginal $\mathrm{pH}$, and reducing atrophic effects on epithelial integrity and vaginal secretions. In addition, treatment with TX-004HR was safe and well tolerated when administered intravaginally once daily for 14 days. Also, as demonstrated in phase 1 pharmacokinetic studies, systemic estrogen levels with TX-004HR were two to three times lower than with an approved vaginal $E_{2}$ tablet at equivalent doses. ${ }^{12}$ Thus, this new vaginal $\mathrm{E}_{2}$ softgel capsule is effective at treating the signs of VVA with low systemic estrogen levels. Furthermore, the results of a survey of the women participating in this study indicate that the majority of women thought the capsule was easy to use, were satisfied with the product, and would consider using the treatment again. $^{15}$

The significant improvements in the signs of VVA that this study found with the TX-004HR vaginal $E_{2}$ softgel capsule were clinically meaningful. The efficacy observed with TX004HR at week 2 with objective markers (ie, changes in VMI) in our study seems to be superior to historical data reported with the vaginal $E_{2}$ tablet at the same dose $\left(10 \mu g E_{2}\right)$. In a 52-week, multicenter, double-blind study $(\mathrm{n}=309)$, improvements were observed from baseline after 2 weeks of daily administration of a $10-\mu \mathrm{g} E_{2}$ tablet that were significantly better than with placebo. The increase in the percentage of superficial cells at 2 weeks was significantly greater than placebo, but was less than the improvement seen in this study in which superficial cells increased to $37 \%$ after 2 weeks of treatment. ${ }^{16}$

The significant improvements seen in our pilot phase 2 study in all components of the VMI, in $\mathrm{pH}$, and in observations of vaginal epithelial integrity and secretions are an important indication of potential improvements in symptoms of VVA. The VMI and $\mathrm{pH}$ are the most useful objective markers of vaginal atrophy due to estrogen decline. ${ }^{17}$ Visual assessments of the vaginal mucosa have been shown to correlate with the objective measures and, thus, are an additional validation of the diagnosis of VVA and of the monitoring of effects of treatment on VVA. ${ }^{18}$ Significant improvements in VMI, $\mathrm{pH}$, and the vaginal examination (vaginal epithelial integrity and vaginal secretions) were observed within 2 weeks. With a longer duration of treatment and follow-up, and a larger sample size, improvements in the vaginal MBS would be expected to follow.

No major or unexpected safety concerns were observed with TX-004HR vaginal $\mathrm{E}_{2}$ softgel capsule treatment during the study period. This is consistent with the favorable safety profiles of other low-dose vaginal estrogen therapies that have been previously reported. ${ }^{19-21}$

The testing of the safety and efficacy of TX-004HR vaginal $\mathrm{E}_{2}$ softgel capsules is currently being expanded in the phase 3 
REJOICE trial. Three doses are being tested $(25 \mu \mathrm{g}, 10 \mu \mathrm{g}$, and an ultra-low-dose of $4 \mu \mathrm{g}$ ) with additional outcome measures. Primary outcome measures include change from baseline in VMI, vaginal $\mathrm{pH}$, and the severity of dyspareunia as MBS after 12 weeks. Secondary outcome measures include change from baseline in the severity of vaginal dryness and vaginal burning; appearance of the vaginal mucosa at 2, 6, 8, and 12 weeks; change from baseline in Female Sexual Function Index at 12 weeks; and hormone concentration assessments at pretreatment, day 1, and weeks 2 and 12 .

\section{CONCLUSIONS}

The results of this study suggest that TX-004HR vaginal $\mathrm{E}_{2}$ softgel capsules are a safe, effective, local, novel treatment option for postmenopausal women with moderate-tosevere VVA. Improvements in vaginal cytology and $\mathrm{pH}$ from baseline at week 2 were significantly higher with TX004HR than with placebo. These improvements on vaginal cell physiology seem to be superior to other vaginal therapies based on historical data. The clinical evidence presented here, coupled with favorable pharmacokinetic results showing two to three times lower systemic exposure of $E_{2}$ than an approved vaginal $\mathrm{E}_{2}$ tablet at the same dose, along with positive satisfaction survey results, is promising and has helped to frame the design of a larger phase 3 trial to further evaluate the safety and clinical efficacy of TX004HR. If approved, the TX-004HR vaginal $\mathrm{E}_{2}$ softgel capsule would provide a new alternative for women with VVA, with better efficacy, earlier onset of action, easier usage, and lower systemic estrogen exposure than currently available options.

Acknowledgments: We thank Chastity Bradley, $\mathrm{PhD}$, and Jolene Mason, PhD, of Precise Publications for their assistance in the writing of this manuscript.

\section{REFERENCES}

1. Versi E, Harvey MA, Cardozo L, Brincat M, Studd JW. Urogenital prolapse and atrophy at menopause: a prevalence study. Int Urogynecol J Pelvic Floor Dysfunct 2001;12:107-110.

2. Stenberg A, Heimer G, Ulmsten U, Cnattingius S. Prevalence of genitourinary and other climacteric symptoms in 61-year-old women. Maturitas 1996;24:31-36.

3. Mac Bride MB, Rhodes DJ, Shuster LT. Vulvovaginal atrophy. Mayo Clin Proc 2010;85:87-94.

4. De Villiers TJ, Pines A, Panay N, et al. Updated 2013 International Menopause Society recommendations on menopausal hormone therapy and preventive strategies for midlife health. Climacteric 2013;16: 316-337.
5. Simon JA, Kokot-Kierepa M, Goldstein J, Nappi RE. Vaginal health in the United States: results from the Vaginal Health: Insights, Views \& Attitudes survey. Menopause 2013;20:1043-1048.

6. Howden LM, Meyer JA. Age and sex composition: 2010 Census Briefs. US Census Bureau. May 2011. Available at: http://www.census.gov/prod/ cen2010/doc/sf1.pdf. Accessed April 14, 2015.

7. Kingsberg SA, Wysocki S, Magnus L, Krychman ML. Vulvar and vaginal atrophy in postmenopausal women: findings from the REVIVE (REal Women's VIews of Treatment Options for Menopausal Vaginal ChangEs) survey. J Sex Med 2013;10:1790-1799.

8. North American Menopause Society. The 2012 hormone therapy position statement of: The North American Menopause Society. Menopause 2012;19:257-271.

9. North American Menopause Society. Management of symptomatic vulvovaginal atrophy: 2013 position statement of The North American Menopause Society. Menopause 2013;20:888-902.

10. Sturdee DW, Panay N. Recommendations for the management of postmenopausal vaginal atrophy. Climacteric 2010;13:509-522.

11. Weisberg E, Ayton R, Darling G, et al. Endometrial and vaginal effects of low-dose estradiol delivered by vaginal ring or vaginal tablet. Climacteric 2005;8:83-92.

12. Pickar JH, Amadio JM, Hill J, Bernick B, Mirkin S. Pilot and pharmacokinetic studies of solubilized estradiol administered vaginally in a softgel capsule. Presented at: the Annual Meeting of the North American Menopause Society; October 15-18, 2014; Washington, DC. Menopause 2014; $21: 1328$ [Abstract S-6].

13. Manson JE, Goldstein SR, Kagan R, et al. Why the product labeling for low-dose vaginal estrogen should be changed. Menopause 2014;21:911916.

14. US Department of Health and Human Services, Food and Drug Administration, and Center for Drug Evaluation and Research. Guidance for industry: labeling guidance for noncontraceptive estrogen drug products for the treatment of vasomotor symptoms and vulvar and vaginal atrophy symptoms-prescribing information for health care providers and patient labeling. Available at: http://www.fda.gov/downloads/ drugs/guidancecomplianceregulatoryinformation/guidances/ucm075090.pdf. Accessed December 2014.

15. Kingsberg S, Amadio J, Graham S, Bernick B, Mirkin S. Patient experience with solubilized estradiol given vaginally in a novel softgel capsule (VagiCap). Presented at: the Annual Meeting of the International Society for the Study of Women's Sexual Health; February 19-22, 2015; Austin, TX. Available at: http://www.isswshmeeting.org/index.php?option= com_content\&view=article\&id=126\&abstract_id $=56$. Accessed February 19, 2015.

16. Simon J, Nachtigall L, Gut R, Lang E, Archer DF, Utian W. Effective treatment of vaginal atrophy with an ultra-low-dose estradiol vaginal tablet. Obstet Gynecol 2008;112:1053-1060.

17. Nilsson K, Risberg B, Heimer G. The vaginal epithelium in the postmenopause-cytology, histology and $\mathrm{pH}$ as methods of assessment. Maturitas 1995;21:51-56.

18. Constantine GD, Kushner H, Bernick B, Graham S, Mirkin S. Vaginal physical examination correlates with vaginal epithelial cells and $\mathrm{pH}$ and can be used to assess treatment efficacy [Presentation FRI-126]. Presented at: Endocrine Society's 97th Annual Meeting and Expo; March 5-8, 2015; San Diego.

19. Bachmann G, Lobo RA, Gut R, Nachtigall L, Notelovitz M. Efficacy of low-dose estradiol vaginal tablets in the treatment of atrophic vaginitis: a randomized controlled trial. Obstet Gynecol 2008;111:67-76.

20. Bachmann G, Bouchard C, Hoppe D, et al. Efficacy and safety of lowdose regimens of conjugated estrogens cream administered vaginally. Menopause 2009;16:719-727.

21. Freedman M, Kaunitz AM, Reape KZ, Hait H, Shu H. Twice-weekly synthetic conjugated estrogens vaginal cream for the treatment of vaginal atrophy. Menopause 2009;16:735-741. 